\title{
Plausible scenario for a generic violation of the weak cosmic censorship conjecture in asymptotically flat four dimensions
}

\author{
Felicity C. Eperon, ${ }^{*}$ Bogdan Ganchev® ${ }^{\dagger}$ and Jorge E. Santos ${ }^{\ddagger}$ \\ Department of Applied Mathematics and Theoretical Physics, University of Cambridge, \\ Wilberforce Road, Cambridge CB3 OWA, United Kingdom
}

(Received 9 December 2019; accepted 4 February 2020; published 28 February 2020)

\begin{abstract}
We present a plausible counterexample to the weak cosmic censorship conjecture in the fourdimensional Einstein-Scalar theory with asymptotically flat boundary conditions. Our setup stems from the analysis of the massive Klein-Gordon equation on a fixed Kerr black hole background. In particular, we construct the quasinormal spectrum numerically, and analytically in the WKB approximation, then go on to compute its backreaction on the Kerr geometry. In the regime of parameters where the analytic and numerical techniques overlap we find perfect agreement. We give strong evidence for the existence of a nonlinear instability at late times.
\end{abstract}

DOI: $10.1103 /$ PhysRevD.101.041502

\section{INTRODUCTION}

Quantum gravity remains terra incognita, largely because it is hard to access experimentally. One might wonder why, since singularities are known to form under a variety of circumstances [1]. However, general relativity stubbornly conceals these regions of high curvature, where quantum gravity is likely to play a leading role, by hiding them behind an absolute event horizon. This phenomenon is observed for such large classes of initial data that it was promoted to a conjecture in [2]. This is the weak cosmic censorship conjecture (WCCC), which forbids the formation of naked singularities, i.e., singularities in causal contact with future null infinity, starting from generic initial data.

Here we propose a four-dimensional asymptotically flat counterexample to the WCCC motivated by the superradiant instability [3-12], afflicting massive scalar perturbations around Kerr black holes (BHs) [13]—rotating, with spherical topology and considered the most general $\mathrm{BH}$ solutions of the vacuum Einstein equation [14].

The angular dependence of such perturbations around Kerr is parametrized by two integers $\{m, \ell\}: m$ counts the number of nodes in the azimuthal direction and $\ell-|m|$ the number of zeros in the polar direction. We show that for any scalar field of mass $\mu$ and any nonzero value of the BH spin, for sufficiently large values of $\ell=m$, these perturbations herald instabilities around the $\mathrm{BH}$, extracting energy and angular momentum. Furthermore, the time scales associated to these instabilities grow parametrically as $e^{4 \ell \log \ell}$, indicating that each of the $\ell$ modes decouples from the rest, evolving independently.

\footnotetext{
fce21@cam.ac.uk bvg25@cam.ac.uk jss55@cam.ac.uk
}

As time progresses, modes with smaller values of $\ell$ stabilize one by one, forming scalar clouds around the $\mathrm{BH}$, similar to those in $[15,16]$. However, these $\mathrm{BHs}$ were shown to be unstable to higher $m$-modes [17], giving rise to the expectation of a cascade towards larger values of $\ell$. This corresponds to a transfer of energy from lower $\ell$-modes to higher ones, indicating an evolution towards smaller scales-a phenomenon akin to turbulence in nonrelativistic $3+1$ fluids.

A possible stabilizing mechanism is the emission of gravitational waves (GWs) by the scalar clouds $[18,19]$. Were they to dissipate energy faster than superradiance creates them, the above scenario would not be possible. We numerically compute the GW emission for fixed gravitational coupling, $M \mu$, and spin parameter, $J / M^{2}$, as a function of $\ell=m$ and find that it leads to energy and angular momentum dispersion that would not be able to counter the efficiency of superradiance.

Our paper is organized as follows: first we present our setup and provide analytic and numerical data for the instability time scales at large $\ell$. We then compute, numerically, the energy radiated towards future null infinity in this process as well as the backreaction of the scalar on one of the components of the Weyl tensor. We see that modes with higher $\ell$ radiate less, implying that energy is accumulated at small scales more efficiently for larger values of $\ell$. Finally we end with discussion of the results.

\section{SETUP}

We work with the Einstein-Hilbert action minimally coupled to a real massive scalar field $\psi$,

$$
S=\int_{\mathcal{M}} \mathrm{d}^{4} x \sqrt{-g}\left(\frac{R}{16 \pi G}-\nabla_{a} \psi \nabla^{a} \psi-\mu^{2} \psi^{2}\right),
$$


where $\mu$ is the scalar field mass, $g_{a b}$ the spacetime metric and $R$ its Ricci scalar. The equations of motion are

$$
R_{a b}-\frac{R}{2} g_{a b}=8 \pi G T_{a b},
$$

where $R_{a b}$ is the Ricci tensor of $g_{a b}$,

$$
\square \psi=\mu^{2} \psi,
$$

and

$$
T_{a b}=2 \nabla_{a} \psi \nabla_{b} \psi-g_{a b} \nabla^{c} \psi \nabla_{c} \psi-\mu^{2} \psi^{2} g_{a b} .
$$

An important solution to these equations is the Kerr $\mathrm{BH}$ [13], with $\psi=0$ and

$$
\begin{aligned}
\mathrm{d} s^{2}= & -\frac{\Delta}{\Sigma^{2}}\left(\mathrm{~d} t-a \sin ^{2} \theta \mathrm{d} \phi\right)^{2}+\Sigma^{2}\left(\mathrm{~d} \theta^{2}+\frac{\mathrm{d} r^{2}}{\Delta}\right) \\
& +\frac{\sin ^{2} \theta}{\Sigma^{2}}\left[a \mathrm{~d} t-\left(r^{2}+a^{2}\right) \mathrm{d} \phi\right]^{2},
\end{aligned}
$$

where $\Delta=r^{2}+a^{2}-2 M r, \Sigma^{2}=r^{2}+a^{2} \cos ^{2} \theta, \phi \in(0,2 \pi)$ is a periodic coordinate and $\theta \in(0, \pi)$ is a polar coordinate. The BH event horizon is a null hypersurface with $r=r_{+} \equiv$ $M+\sqrt{M^{2}-a^{2}}$, angular velocity $\Omega_{K}=a /\left(a^{2}+r_{+}^{2}\right)$ and surface gravity $\kappa_{K}=\left(r_{+}^{2}-a^{2}\right) /\left[2 r_{+}\left(r_{+}^{2}+a^{2}\right)\right]$. The constant $M$ is the $\mathrm{BH}$ mass, and $a$ parametrizes its angular momentum via $J=M a$. The absence of naked singularities demands $|a| \leq M$ with the inequality being saturated at extremality, when the Kerr BH event horizon becomes degenerate with $\kappa_{K}=0$.

We study Eq. (2b) on a fixed Kerr background (3), which is stationary and axisymmetric with respect to the Killing fields $\partial / \partial t$ and $\partial / \partial \phi$, respectively. We consider perturbations of the form,

$$
\psi(t, r, \theta, \phi)=e^{-i \omega t+i m \phi} \hat{\psi}_{\omega m}(r, \theta)
$$

and assume that $\hat{\psi}_{\omega m}(r, \theta)$ is separable, $\hat{\psi}_{\omega m}(r, \theta)=$ $R_{\omega \ell m}(r) S_{\omega \ell m}(\theta)$, with the label $\ell$ anticipating that the separation constant will be parametrized by an integer $\ell$. Not all solutions to Eq. (2b) are separable, but we are interested in those composed of the sum (possibly infinite) of such separable solutions. $\omega \in \mathbb{C}$ is a complex frequency, determined by imposing appropriate boundary conditions. We are interested in finding unstable mode solutions for which $\operatorname{Im}(\omega)>0$.

Inserting the ansatz (4) into Eq. (2b) yields a system of two second order ordinary differential equations, coupled via the separation constant $\Lambda$,

$$
\Delta\left[\Delta R_{\omega \ell m, r}\right]_{, r}+V(r) R_{\omega \ell m}=0,
$$

$$
\frac{1}{\sin \theta}\left[\sin \theta S_{\omega \ell m, \theta}\right]_{, \theta}-\left[a^{2} k^{2} \cos ^{2} \theta+\frac{m^{2}}{\sin ^{2} \theta}-\Lambda\right] S_{\omega \ell m}=0,
$$

where

$$
\begin{aligned}
V(r)= & -k^{2} r^{4}+2 M \mu^{2} r^{3}-\left(\Lambda+a^{2} k^{2}\right) r^{2} \\
& +\left(2 M \Lambda-4 a m M \omega+2 M a^{2} \omega^{2}\right) r-a^{2}\left(\Lambda-m^{2}\right)
\end{aligned}
$$

with $k \equiv \sqrt{\mu^{2}-\omega^{2}}$. Finding unstable modes amounts to finding the values of $\omega$ for which $\psi$ has ingoing boundary conditions at the event horizon (consistent with the equivalence principle) and finite energy on a partial Cauchy surface $t=$ const.

This problem can be tackled numerically (for any values of the parameters) and analytically (in certain regions of moduli space). We will first compute the modes using a WKB expansion in $m$, which we detail next.

\section{WKB EXPANSION AND NUMERICAL VALIDATION}

Our WKB expansion is valid for any spin parameter $|a|<M$, and only assumes $m$ to be large. For small values of $a$ and $\mu$, it reproduces the results in [7,20] (up to a factor of 2, see [21]). For large $m$, Eq. (5b) can be approximated by the usual equation for spherical harmonics on a 2-sphere so that $\Lambda=\ell(\ell+1)+O\left(\ell^{-1}\right)$ and $\ell, m$ are integers with $\ell \geq 0,|m| \leq \ell$. In the following we set $m=\ell$ and take the limit $\ell \gg 1$.

To determine the large $\ell$ limit at fixed $\mu$ and $a$, we used the same method as in [22], which combines a matched asymptotic and WKB type approach. We quote the final result and leave the details to the Supplemental Material [23],

$$
\begin{aligned}
& \operatorname{Re}(\omega M)=\hat{\mu}\left(1-\frac{\hat{\mu}^{2}}{2 \ell^{2}}\right)+\mathcal{O}\left(\ell^{-3}\right), \\
\operatorname{Im}(\omega M)= & \frac{\ell^{-4 \ell-\frac{9}{2}+p}}{2^{2 \ell+1-p} \sqrt{\pi} p !} \hat{\mu}^{4 \ell+5} \sinh \left[\frac{\pi\left(\ell \Omega_{K}-\mu\right)}{\kappa_{K}}\right] \\
& \times \exp \left[-\frac{2}{\kappa_{K}}\left(\ell \Omega_{K}-\frac{\hat{\mu}}{r_{+}}\right) \arctan \left(\frac{\Omega_{K}}{\kappa_{K}}\right)\right. \\
& -2(1-\ell+p)] \times\left[1+\mathcal{O}\left(\ell^{-1}\right)\right],
\end{aligned}
$$

where $\hat{\mu} \equiv \mu M$ and $p \in \mathbb{N}_{0}$ is a radial overtone. First note that if we set $a=0$, the argument of the sinh becomes negative, and the instability disappears. Furthermore, its onset sits precisely at the onset of superradiance, namely $\ell \Omega_{K}=\operatorname{Re}(\omega)$. More importantly for our purposes, in the 


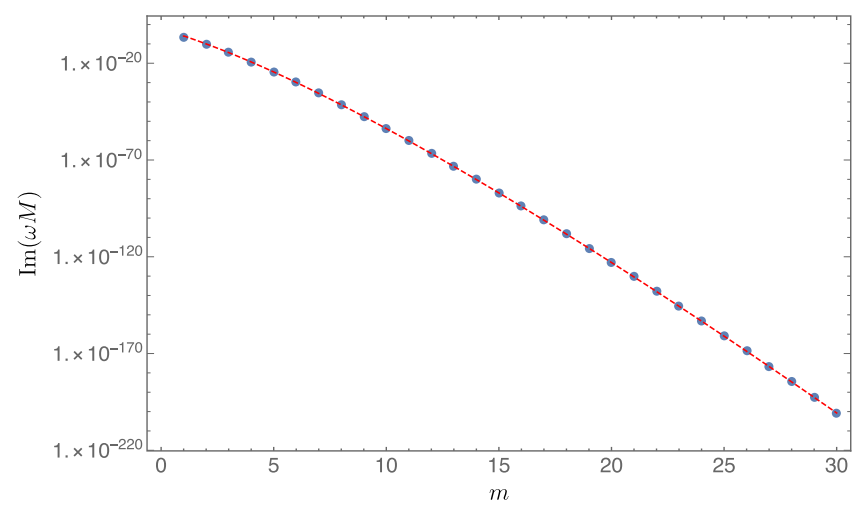

FIG. 1. The superradiant modes of a massive scalar around Kerr with $M \mu=0.42$ and $J / M^{2}=0.99$, as a function of $m$. The dashed red curve shows the analytic expression (6b), and the blue disks our exact numerical data.

limit $\ell \rightarrow+\infty$, the growth rate of the instability scales as $e^{-4 \ell \log \ell}$, and no matter what the value of $\mu$ or $a$, one can always find a value of $\ell=\ell_{\star} \equiv\left\lceil\mu / \Omega_{K}\right\rceil$ above which the instability sets in. This shows that all Kerr black holes are unstable to massive scalar field perturbations, irrespective of their initial spin $|J|<M^{2}$ and of the mass $\mu$ of the scalar perturbation.

One can test the regime of validity of our approximation, by comparing with data obtained by solving numerically, without approximations, the full equations. To this end, we perform a change of variables so that we only solve for smooth functions in their integration domain. This necessarily involves a choice of normalization, which we describe next. For numerical aid we define

$$
\begin{gathered}
R_{\omega \ell m}(r)=\left(1-\frac{r_{+}}{r}\right)^{-i \frac{\omega-m \Omega_{K}}{2 \kappa_{K}}} e^{-k r}\left(\frac{r_{+}}{r}\right)^{\gamma} q_{r}(r), \\
S_{\omega \ell m}(\theta)=\sin ^{m} \theta q_{\theta}(\theta)
\end{gathered}
$$

with $q_{r}\left(r_{+}\right)=q_{\theta}(0)=1$ and $\gamma \equiv 1+2 M k-M \mu^{2} / k$. We now use the methods of [24] to solve for the eigenpair $(\omega, \Lambda)$ using a Newton-Raphson routine [25].

As seen in Fig. 1, our numerical data agrees excellently with (6b). Furthermore, one can measure deviations of our WKB expression to the exact numerical result, and it agrees with the error given in Eq. (6b).

\section{BACKREACTION}

We want the GWs emitted by a scalar cloud around a Kerr $\mathrm{BH}$ and its leading order backreaction on the geometry. In the vector field case $[26,27]$ it has been shown that the system evolves adiabatically; the emergence of the cloud due to superradiance, and the consecutive saturation of the vector mode responsible, due to the spinning down of the $\mathrm{BH}$, proceed on a much faster time scale than the dispersion of energy and angular momentum due to $\mathrm{GW}$ emission from the cloud.

We proceed using nonlinear perturbation theory and declare

$\psi=\sum_{i=0}^{+\infty} \psi^{(2 i+1)} \varepsilon^{2 i+1}, \quad$ and $\quad g=g_{K}+\sum_{i=1}^{+\infty} g^{(2 i)} \varepsilon^{2 i}$,

where $g_{K}$ is given by the Kerr metric (3). We expand the equations of motion (2) in a power series in $\varepsilon$. To first order in $\varepsilon$ we solve Eq. (2b) subject to a choice of initial data. For the case at hand, we choose $\psi$ to be given by the real part of one of the unstable modes we have determined above. These are labeled by a given value of $m$. Furthermore, since $\operatorname{Im}(\omega M) \ll \operatorname{Re}(\omega M)$, we take $\omega$ to be purely real. We then proceed to second order and attempt to compute the leading order backreaction on the metric, $g^{(2)}$, and its associated curvature. The standard approach to the linearized Einstein equation presents us with a daunting task; however, Kerr BHs are algebraically special, allowing us to bypass computing $g^{(2)}$, and directly calculate certain gauge invariant scalars built out of the Weyl tensor. These do not couple amongst themselves, and we focus on the Newman-Penrose scalar $\psi_{4}$ since it also allows us to efficiently compute the GWs emitted by the scalar cloud. $\psi_{4}$ obeys the Teukolsky equation [28-30],

$$
\begin{aligned}
& {\left[(\Delta+3 \gamma-\bar{\gamma}+4 \mu+\bar{\mu})(D+4 \epsilon-\rho)-3 \psi_{2}\right.} \\
& \quad-(\bar{\delta}+3 \alpha+\bar{\beta}+4 \pi-\bar{\tau})(\delta+4 \beta-\tau)] \psi_{4}=4 \pi T_{4},
\end{aligned}
$$

whereby all quantities appearing in Eq. (9) are given in the Supplemental Material [23]. The source term $T_{4}$ is reconstructed directly from Eq. (2c) and thus depends on $\psi^{(1)}$ and its gradient only.

The lhs of (9) can be separated into angular and radial parts as in the vacuum case [28], allowing us to solve for $\psi_{4}$ as an infinite sum of separable solutions using Green's method.

From $\psi_{4}$, Teukolsky [30] showed us how to compute the rate of gravitational radiation at future null infinity,

$$
\frac{\mathrm{d}^{2} E_{s}}{\mathrm{~d} t \mathrm{~d} \Omega}=\lim _{r \rightarrow \infty} \frac{r^{2}}{4 \pi \hat{\omega}^{2}}\left|\psi_{4}\right|^{2},
$$

where $\hat{\omega}=2 \omega$ and $\mathrm{d} \Omega$ is the induced volume on a unit 2-sphere.

We work with the scaled expression,

$$
P_{E}=\frac{\mathrm{d} E_{s}}{\mathrm{~d} t}\left(\frac{M}{\mathcal{M}_{s}}\right)^{2},
$$

where 


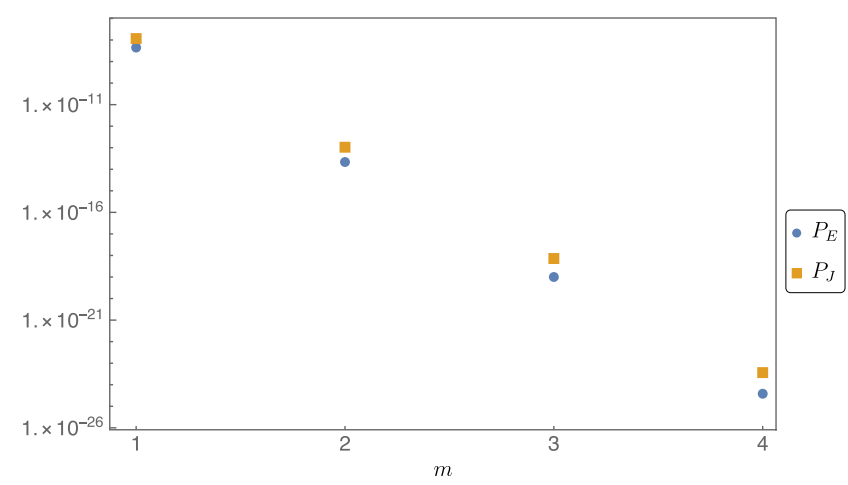

FIG. 2. GW emission of energy and angular momentum, $P_{E}$ and $P_{J}$ respectively, for a single $\ell=m$ scalar cloud around Kerr as a function of $m$. Same parameters as in Fig. 1.

$$
\mathcal{M}_{s}=\int_{r_{+}}^{+\infty} \int_{0}^{\pi} \int_{0}^{2 \pi} \sqrt{-g} T^{t}{ }_{t} \mathrm{~d} \phi \mathrm{d} \theta \mathrm{d} r
$$

is the total scalar field energy, - the energy of the perturbed initial data. $P_{E}$ is independent of the scalar field amplitude and measures the energy radiated per $m$ mode in the initial data. See the Supplemental Material [23] on how to compute $P_{E}$.

As a measure of curvature, we monitored the following time independent quantity as a function of $m$ :

$$
\chi \equiv \max _{r, \theta}\left(\left|\psi_{4}\right|^{2} / \mathcal{M}_{S}^{2}\right)
$$

The radial and azimuthal location of the maximum of (13), $(r, \theta)=\left(r_{\star}, \theta_{\star}\right)$, track the maximum of $\left[\psi^{(1)}\right]^{2}$.

Our results for the GW emission are shown in Fig. 2. The radiated angular momenta in this process is $P_{J}=\frac{m}{\operatorname{Re}(\omega M)} P_{E}$, in accordance with [30]. The fact that both $P_{E}$ and $P_{J}$ appear to decrease rapidly with increasing $m$ shows that the evolution occurs, to very good approximation, at fixed energy and angular momentum. This is akin to the time evolution of the superradiance instability with anti-de Sitter asymptotics [31,32], simulated recently in [33], and shows hints of turbulent behavior.

The data in Fig. 2 is for a fixed value of the dimensionless spin parameter $a / M$. However, during the aforementioned cascade the $\mathrm{BH}$ will be gradually spinning down; hence the gravitational radiation for each value of $m$ should ideally be computed by accounting for BH's loss of energy due to the superradiant modes active prior to the one under consideration. Nevertheless, using the superradiant condition $\operatorname{Re}(\omega)>m \Omega_{K}$, one sees that $\Delta(a / M)$ for successive superradiant modes $\sim \ell^{-1}$ as $\ell \rightarrow \infty$, implying that in the regime of interest the dimensionless spin will be approximately constant and Fig. 2 represents accurately the qualitative behavior.

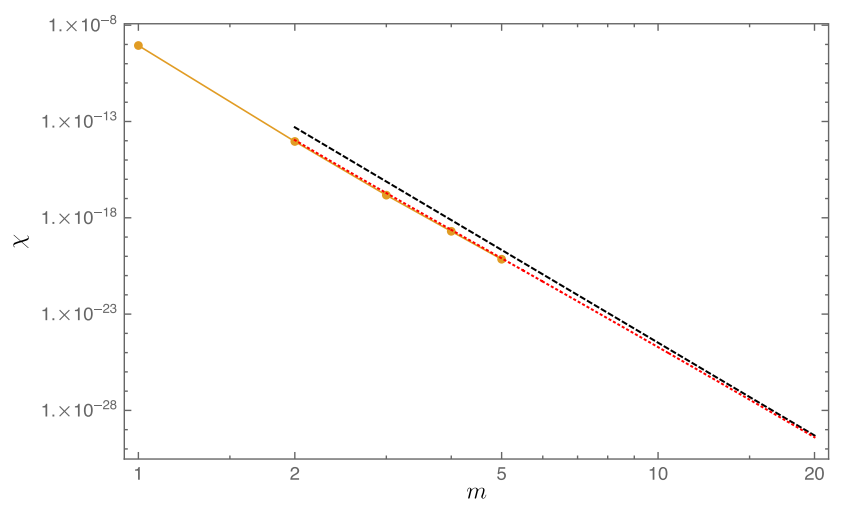

FIG. 3. $\chi$, a measure of the spacetime curvature, as a function of $\ell=m$. Same parameters as in Fig. 1. The black dashed curve shows our leading order approximation, whereas the dotted red line includes the next to leading order correction and the orange circles are numerical data.

In Fig. 3 we show the dependence of $\chi$ on the initial data, here labeled by $m$. A WKB-type analysis reveals that the leading behavior for $\chi$ is power law in $1 / m$,

$$
\chi_{\mathrm{WKB}}=\frac{\hat{\mu}^{10}}{m^{16} \pi^{2} 2^{6}}\left(\frac{\mu}{r_{+}}\right)^{6} \text {. }
$$

One can consistently correct this approximation to next-toleading order (see the Supplemental Material [23]).

Note that $P_{E}$ is harder to compute numerically than $\chi$, hence why we have extended results for $\chi$ up to $m=5$.

\section{GEDANKEN EXPERIMENT}

We are now ready to present our possible counterexample to WCCC with asymptotically flat boundary conditions. Consider generic initial data for the Einstein-Scalar system. These data are controlled by a large functional freedom coming from the fact that we can choose the initial metric on a constant time slice, as well as the extrinsic curvature (so long as the Hamiltonian and momentum constraints are satisfied). In addition, we can also control the initial profile for the scalar field and its first time derivative on a constant time slice. We are going to choose our initial data to be close to that of the Kerr BH, so that deviations from the Kerr metric only occur at order $\mathcal{O}\left(\psi^{2}\right)$. This condition can be relaxed by considering initial data for the purely gravitational sector that is small in some norm. This essentially means that all the dynamics are being generated by the scalar field.

For generic scalar field initial data, we expect the scalar field profile to have some support on the unstable modes of the preceding sections, i.e., to excite unstable modes. Since all other modes decay with time [34], we expect the late time evolution to be dominated by the leading unstable modes and their backreaction. For each value of $m$ there is an infinite number of such modes labeled by $\ell \geq|m|$. 
However, all of these modes stop being unstable as soon as the condition $\operatorname{Re}(\omega)>m \Omega_{K}$ is no longer satisfied. The dynamics of this change in angular momentum and energy is entirely controlled by the $\ell=m$ modes, implying that, after some suitably long time, the dynamics of the EinsteinScalar system can be well approximated by restricting our attention to scalar profiles of the form,

$\psi(t, r, \theta, \phi)=\operatorname{Re}\left[\sum_{\ell=0}^{+\infty} a_{\ell} e^{-i \omega_{\ell}+i \ell \phi} R_{\omega_{\ell} \ell \ell}(r) S_{\omega_{\ell} \ell \ell}(\theta)\right]$,

and determining its leading order backreaction on the spacetime curvature. The coefficients $a_{\ell}$ are determined by our choice of initial data: for finite Sobolev norm initial data we expect $a_{\ell}$ to exhibit polynomial behavior in $1 / \ell$, whereas for $C^{\infty}$ initial data we expect the coefficients $a_{\ell}$ to decay faster than any polynomial in $1 / \ell$. Note that for real analytic initial data one can show that $a_{\ell} \approx e^{-\alpha \ell}$, for $\alpha>0$.

Given that each $\ell$ mode evolves on an exponentially different timescale, as shown by Eq. (6b), they effectively decouple from each other, allowing us to study each term in Eq. (15) and its backreaction on the metric separately. Eventually, a given $\ell=\ell_{\star}$ mode becomes stable, but the system remains unstable to higher values of $\ell>\ell_{\star}$. This cascading happens slowly, since the time scales for this effect are exponentially large. One might worry that the energy contained in these high $\ell$ modes is radiated away as time passes by, but we have seen in Fig. 2 that this is not the case. In fact, the larger the value of $\ell$, the smaller its radiative power is.

Finally, we have seen in Fig. 3 that $\chi$ decays as $\ell^{-16}$. This in turn implies that a mode with weigh $a_{\ell}$ will depend on $\ell$ as $a_{\ell}^{4} \ell^{-16}$. The reason for this is simple: the Teukolsky scalar $\psi_{4}$ is sourced by $\left[\psi^{(1)}\right]^{2}$, and $\chi$ is related to $\left|\psi_{4}\right|^{2}$, which translates into the overall scaling mentioned above. The curvatures are thus suppressed with time, and the evolution continues until all of the angular momentum is deposited into the scalar cloud, and the central black hole becomes Schwarzschild. However, one can show [35] that massive scalar field perturbations decay extremely slow around Schwarschild black holes, with a dependence as weak as $1 / \log (\log t)$, for large $t$. This suggests that the hypothetical end point is itself nonlinearly unstable through a mechanism similar to the one reported in $[22,36,37]$. On the other hand, this slow decay warrants the question whether the gravitational radiation might not just disperse the clouds, before any nonlinearities become problematic. We cannot answer this in our analysis, as our approximation for radiation emission breaks down at late times. Therefore the outcome of our thought experiment depends on which of the competing processes - the GW emission or the nonlinear issues resulting from slower than logarithmic decay-wins over. If it is the latter, then the lack of a possible stationary end point leads us to conjecture that the spirit, if not the letter, of weak cosmic censorship is violated. Whether the curvature will be infinite in finite time is a question that we cannot settle with our current methods.

Note also that $r_{\star}(\ell)$ increases with $\ell$, posing a problem from a numerical perspective: 1) the time scales involved are enormous; 2) the cascading towards high $\ell$ values makes this problem dependent on high frequency modes (as the simulation of turbulence in $3+1$ nonrelativitic fluids); and 3) the integration domain must extend to spatial infinity to observe this effect.

\section{CONCLUSIONS}

We have seen (Fig. 2) that the efficiency of superradiance cannot be counteracted by GW emission, implying that the system will continue advancing to higher values of $m$ with curvatures decreasing appropriately (as shown by Fig. 3) until a configuration with a central Schwarzschild black hole is reached. However, Schwarzschild is likely to be nonlinearly unstable due to the very slow decay of perturbations induced by massive scalar fields. Reaching this troublesome regime, given that effects we cannot account for in our analysis do not prevent this, will involve time scales much longer than the age of our Universe, of course, as one will have to go to large values of $\ell=m$. Nevertheless our scenario provides the first plausible example of a system with asymptotically flat boundary conditions, where WCCC is violated.

\section{ACKNOWLEDGMENTS}

We would like to thank O. J. C. Dias and H. S. Reall for reading an earlier version of this manuscript. We also want to thank W. E. East for many useful discussions that led to this paper. F. C. E. and B. G. are supported by STFC. J. E. S is supported in part by STFC Grants No. PHY-1504541 and No. ST/P000681/1. This work used the DIRAC Shared Memory Processing system at the University of Cambridge, operated by the COSMOS Project at the Department of Applied Mathematics and Theoretical Physics on behalf of the STFC DiRAC HPC Facility (www.dirac.ac.uk). This equipment was funded by BIS National E-infrastructure Capital Grant No. ST/J005673/1, STFC Capital Grant No. ST/H008586/1, and STFC DiRAC Operations Grant No. ST/K00333X/1. DiRAC is part of the National e-Infrastructure. 
[1] J. M. M. Senovilla, Singularity theorems and their consequences, Gen. Relativ. Gravit. 30, 701 (1998).

[2] R. Penrose, Gravitational collapse: The role of general relativity, Riv. Nuovo Cimento 1, 252 (1969); Gen. Relativ. Gravit. 34, 1141 (2002).

[3] J. M. Bardeen, W. H. Press, and S. A. Teukolsky, Rotating black holes: Locally nonrotating frames, energy extraction, and scalar synchrotron radiation, Astrophys. J. 178, 347 (1972).

[4] A. A. Starobinsky, Amplification of waves reflected from a rotating "black hole", Zh. Eksp. Teor. Fiz. 64, 48 (1973) [Sov. Phys. JETP, 37, 28 (1973)].

[5] T. Damour, N. Deruelle, and R. Ruffini, On quantum resonances in stationary geometries, Lett. Nuovo Cimento 15, 257 (1976).

[6] T. J. M. Zouros and D. M. Eardley, Instabilities of massive scalar perturbations of a rotating black hole, Ann. Phys. (N.Y.) 118, 139 (1979).

[7] S. L. Detweiler, Klein-Gordon equation and rotating black holes, Phys. Rev. D 22, 2323 (1980).

[8] S. R. Dolan, Instability of the massive Klein-Gordon field on the Kerr spacetime, Phys. Rev. D 76, 084001 (2007).

[9] H. Yoshino and H. Kodama, Gravitational radiation from an axion cloud around a black hole: Superradiant phase, Prog. Theor. Exp. Phys. 2014, $043 E 02$ (2014).

[10] R. Brito, V. Cardoso, and P. Pani, Black holes as particle detectors: Evolution of superradiant instabilities, Classical Quantum Gravity 32, 134001 (2015).

[11] R. Brito, V. Cardoso, and P. Pani, Superradiance, Lect. Notes Phys. 906, 1 (2015).

[12] Y. Shlapentokh-Rothman, Exponentially growing finite energy solutions for the Klein-Gordon equation on sub-extremal Kerr spacetimes, Commun. Math. Phys. 329, 859 (2014).

[13] R. P. Kerr, Gravitational Field of a Spinning Mass as an Example of Algebraically Special Metrics, Phys. Rev. Lett. 11, 237 (1963).

[14] D. Robinson, Four decades of black holes uniqueness theorems, in Kerr Fest: Black Holes in Astrophysics, General Relativity and Quantum Gravity Christchurch, New Zealand, 2004 (2004).

[15] O. Chodosh and Y. Shlapentokh-Rothman, Time-Periodic Einstein-Klein-Gordon Bifurcations of Kerr, Commun. Math. Phys. 356, 1155 (2017).

[16] C. A. R. Herdeiro and E. Radu, Kerr Black Holes with Scalar Hair, Phys. Rev. Lett. 112, 221101 (2014).

[17] B. Ganchev and J. E. Santos, Scalar Hairy Black Holes in Four Dimensions are Unstable, Phys. Rev. Lett. 120, 171101 (2018).

[18] M. Sasaki and H. Tagoshi, Analytic black hole perturbation approach to gravitational radiation, Living Rev. Relativity $\mathbf{6}$, 6 (2003).

[19] R. Brito, S. Ghosh, E. Barausse, E. Berti, V. Cardoso, I. Dvorkin, A. Klein, and P. Pani, Gravitational wave searches for ultralight bosons with LIGO and LISA, Phys. Rev. D 96, 064050 (2017).

[20] The large $m$ limit does not commute with the extremal limit. It can be shown that at any finite value of $m, \operatorname{Im}(\omega)=0$ at extremality. However, the region of the black hole moduli space where this behaviour occurs scales inversely with $m$, and is thus absent in the strict $m \rightarrow+\infty$ limit. The behaviour in this region could be investigated by switching the order of limits.

[21] H. Furuhashi and Y. Nambu, Instability of massive scalar fields in Kerr-Newman space-time, Prog. Theor. Phys. 112, 983 (2004).

[22] F. C. Eperon, H. S. Reall, and J. E. Santos, Instability of supersymmetric microstate geometries, J. High Energy Phys. 10 (2016) 031.

[23] See Supplemental Material at http://link.aps.org/ supplemental/10.1103/PhysRevD.101.041502 for discussions on our WKB approximation, boundary conditions, numerical convergence tests, details of the projection of Teukolsky equation on a basis of spin-weighted spheroidal harmonics and its analytical approximation.

[24] Ó. J. C. Dias, J. E. Santos, and B. Way, Numerical methods for finding stationary gravitational solutions, Classical Quantum Gravity 33, 133001 (2016).

[25] The only additional complication is that we are searching for extremely small growth rates when $m$ increases, so using extended precision is mandatory.

[26] W. E. East and F. Pretorius, Superradiant Instability and Backreaction of Massive Vector Fields around Kerr Black Holes, Phys. Rev. Lett. 119, 041101 (2017).

[27] W. E. East, Superradiant instability of massive vector fields around spinning black holes in the relativistic regime, Phys. Rev. D 96, 024004 (2017).

[28] S. A. Teukolsky, Perturbations of a rotating black hole. 1. Fundamental equations for gravitational electromagnetic and neutrino field perturbations, Astrophys. J. 185, 635 (1973).

[29] W. H. Press and S. A. Teukolsky, Perturbations of a rotating black hole. II. Dynamical stability of the Kerr metric, Astrophys. J. 185, 649 (1973).

[30] S. A. Teukolsky and W. H. Press, Perturbations of a rotating black hole. III-Interaction of the hole with gravitational and electromagnet ic radiation, Astrophys. J. 193, 443 (1974).

[31] O. J. C. Dias, G. T. Horowitz, and J. E. Santos, Black holes with only one Killing field, J. High Energy Phys. 07 (2011) 115 .

[32] B. E. Niehoff, J. E. Santos, and B. Way, Towards a violation of cosmic censorship, Classical Quantum Gravity 33, 185012 (2016).

[33] P. M. Chesler and D. A. Lowe, Nonlinear Evolution of the $\mathrm{AdS}_{4}$ Superradiant Instability, Phys. Rev. Lett. 122, 181101 (2019).

[34] This decay can be very complicated to determine and is not exponential with time.

[35] Ó. J. C. Dias, J. E. Santos, H. S. Reall, and B. Ganchev (to be published).

[36] J. Keir, Slowly decaying waves on spherically symmetric spacetimes and ultracompact neutron stars, Classical Quantum Gravity 33, 135009 (2016).

[37] J. Keir, Wave propagation on microstate geometries, Ann. Inst. Henri Poincaré 21, 705 (2016). 